\title{
Fixing the non-relativistic expansion of the 1PM potential
}

\author{
Gianluca Grignani, ${ }^{a, b}$ Troels Harmark, ${ }^{c}$ Marta Orselli ${ }^{a, b, c}$ and Andrea Placidi ${ }^{a, b, c}$ \\ ${ }^{a}$ Dipartimento di Fisica e Geologia, Università di Perugia, \\ Via A. Pascoli, 06123 Perugia, Italia \\ ${ }^{b}$ INFN - Sezione di Perugia, \\ Via A. Pascoli, 06123 Perugia, Italia \\ ${ }^{c}$ Niels Bohr Institute, Copenhagen University, \\ Blegdamsvej 17, DK-2100 Copenhagen Ø, Denmark \\ E-mail: gianluca.grignani@unipg.it, harmark@nbi.ku.dk, \\ orselli@nbi.dk , andrea.placidi@studenti.unipg.it
}

ABSTRACT: We obtain a first order post-Minkowskian two-body effective potential whose post-Newtonian expansion directly reproduces the Einstein-Infeld-Hoffmann potential. Post-Minkowskian potentials can be extracted from on-shell scattering amplitudes in a quantum field theory of scalar matter coupled to gravity. Previously, such potentials did not reproduce the Einstein-Infeld-Hoffmann potential without employing a suitable canonical transformation. In this work, we resolve this issue by obtaining a new expression for the first-order post-Minkowskian potential. This is accomplished by exploiting the reference frame dependence that arises in the scattering amplitude computation. Finally, as a check on our result, we demonstrate that our new potential gives the correct scattering angle.

Keywords: Black Holes, Classical Theories of Gravity

ArXiv EPrint: 2006.13234 


\section{Contents}

1 Introduction 1

2 Post-Minkowskian potential 2

2.1 The 1PM potential 5

$\begin{array}{lll}2.2 & \text { Issues with the NR limit of the PM potential } & 7\end{array}$

$\begin{array}{lll}3 & \text { A new expression for the 1PM potential } & 7\end{array}$

4 NR limit beyond the 1PN order $\quad 10$

$5 \quad 1 P M$ scattering angle with the new potential 11

$\begin{array}{ll}5.1 \text { The scattering angle } & 11\end{array}$

6 Conclusion $\quad 14$

$\begin{array}{ll}\text { A The function } A & 14\end{array}$

\section{Introduction}

The calculation of two-body effective potentials for the conservative dynamics of binary systems from on-shell scattering amplitudes is, by now, a well established technique. After its debut [1] in the Post-Newtonian (PN) perturbative framework of General Relativity [2], this method has been mostly employed in the context of the Post-Minkowskian (PM) expansion [3-6]. Indeed, since this technique does not require a non-relativistic (NR) expansion on the typical velocity of the system $v$, a Post-Minkowskian approach where the Newton's constant $G$ is identified as the one and only perturbative parameter of the theory, allows us to obtain the most comprehensive two-body interaction potential that we could build from scattering amplitudes.

In dealing with gravitationally bound states like compact binaries, the virial theorem tells us that kinetic and potential energies must share the same order of magnitude, i.e.

$$
\frac{G M}{r c^{2}} \sim \frac{v^{2}}{c^{2}}
$$

$M$ being the total mass of the system. Therefore, there is no straightforward way to determine whether keeping all order in velocity would effectively bring an increase in accuracy. Nevertheless, PM results for the conservative two-body dynamics have proved to be quite promising [7], both as an input for effective one-body models and as a mean to crosscheck and supplement the preexisting PN results. Specifically in view of the latter purpose, it is important to elucidate how the PM potentials behave once non-relativistically expanded, especially if we want to extract out of them the corresponding terms in the PN expansion. 
With the intention of clarifying this aspect of PM calculations from scattering amplitudes in the simplest possible conditions, this work will be focused on the NR limit of the first order PM potential.

We will start by briefly reviewing how PM potentials are obtained in general, pointing out the mismatch between the NR limit of present 1PM potentials and the associated PN results. Then we will show how the reference frame dependence of the potential can be exploited a priori to find a new expression for the 1PM potential that, once expanded for small velocities, directly reproduces the well-known Einstein-Infeld-Hoffmann 1PN potential [8]. Extending this nice feature to higher PN orders seems a more challenging task and we leave it for future work. Here we will instead present the a posteriori canonical transform that allow us to reproduce the $2 \mathrm{PN}$ order, while genarally setting up the procedure that should be used to do the same for higher PN orders. Finally we will check the physical consistency of our new 1PM potential against the ones already present in the literature, by showing that from it one can obtain the correct scattering angle (see for example $[5,9,10]$ ).

The computation of the scattering angle for non-relativistic quantum mechanical Hamiltonians has a long history. Typically, the interest has been mainly focused on finding approximate (semi-classical) solutions, first through the WKB-approximation, later by considering the eikonal limit (see, e.g., refs. [11-13]). Amplitudes methods have been used more recently to compute scattering angles [10] and we will adopt here the approach of ref. [14].

In this paper, following the vast majority of works on this subject, we leave aside all the finite-size ${ }^{1}$ and spin effects, ${ }^{2}$ that could fit the proper description of some astrophysical binary systems.

\section{Post-Minkowskian potential}

Within the PM scheme, the general two-body effective Hamiltonian for a system of two compact objects gravitationally bound to each other, in the point-particle approximation can be written as

$$
H_{\mathrm{PM}}\left(\boldsymbol{p}_{1}, \boldsymbol{p}_{2}, \boldsymbol{r}\right)=\sqrt{\boldsymbol{p}_{1} c^{2}+m_{1}^{2} c^{4}}+\sqrt{\boldsymbol{p}_{2} c^{2}+m_{2}^{2} c^{4}}+\sum_{n=1}^{+\infty} V_{n \mathrm{PM}}\left(\boldsymbol{p}_{1}, \boldsymbol{p}_{2}, \boldsymbol{r}\right),
$$

where an effective gravitational potential organized in a PM expansion (i.e. in powers of $G$ ) is added to the free-particle energies. Momenta and masses of the two objects are labeled as $\boldsymbol{p}_{1,2}$ and $m_{1,2}$ while $\boldsymbol{r} \equiv \boldsymbol{x}_{1}-\boldsymbol{x}_{2}$ stands for their relative separation. In general, the Hamiltonian underlying the two-body effective theory at hand is not uniquely determined. We are indeed free to perform any canonical transformation we want on (2.1) and thus find different, yet equivalent, expressions for it. At the 1PM order and selecting the center of mass reference frame ${ }^{3}$ we get

$$
H_{1 \mathrm{PM}}(\boldsymbol{p}, \boldsymbol{r})=\sqrt{\boldsymbol{p} c^{2}+m_{1}^{2} c^{4}}+\sqrt{\boldsymbol{p} c^{2}+m_{2}^{2} c^{4}}+V_{1 \mathrm{PM}}(\boldsymbol{p}, \boldsymbol{r}),
$$

\footnotetext{
${ }^{1}$ See ref. [15] and the recent ref. [16] for works on how to include finite-size effects.

${ }^{2}$ See refs. $[17,18]$ for recent attempts to include the spin.

${ }^{3}$ This shift of reference frame amounts indeed to a canonical transformation.
} 
with $\boldsymbol{p} \equiv \boldsymbol{p}_{1}=-\boldsymbol{p}_{2}$. The interaction potential represents the only non-trivial part of the Hamiltonian. Its determination at any given PM order can be accomplished within the framework of on-shell scattering amplitudes [3-6] and we will now describe the main features of this derivation.

The underlying theory, upon which the whole method is based, is a quantum field theory of gravity where in general gravitons are coupled to quantum fields whose degrees of freedom reflect the properties of the systems we wish to describe. In particular, the standard choice for the action is [3]

$$
\mathcal{S}=\int d^{4} x \sqrt{-g}\left[\frac{R}{16 \pi G}+\frac{1}{2} \sum_{a=1,2}\left(g^{\mu \nu} \partial_{\mu} \phi_{a} \partial_{\nu} \phi_{a}-m_{a}^{2} \phi_{a}^{2}\right)\right] .
$$

Here the pure gravity sector can be limited to the Einstein-Hilbert term without renormalizability concerns, since the classical, low energy physics we are interested in is unaffected by higher order terms in the curvature. The remaining parts introduce a minimal gravitymatter coupling in terms of two real scalar fields $\phi_{1,2}$, with masses $m_{1,2}$ corresponding to the two astrophysical objects we want to describe. The minimal coupling relies on the pointparticle approximation and is effectively meaningful only when dealing with really compact objects like black holes. ${ }^{4}$ Moreover, the fact that we work with scalar fields specializes our description to compact binaries whose black holes are Schwarzschild black holes. ${ }^{5}$

As for the metric, the standard quantization scheme involves a perturbative expansion around the flat Minkowskian metric $\eta_{\mu \nu}$, namely

$$
\begin{aligned}
& g_{\mu \nu}=\eta_{\mu \nu}+\sqrt{32 \pi G} h_{\mu \nu}, \\
& g^{\mu \nu}=\eta^{\mu \nu}-\sqrt{32 \pi G} h^{\mu \nu}+32 \pi G h^{\mu \rho} h_{\rho}{ }^{\nu}+O\left(\sqrt{G^{3}}\right),
\end{aligned}
$$

where the small fluctuation $h_{\mu \nu}$ is identified as the graviton field.

After a gauge fixing choice, which is typically the De Donder gauge corresponding to adding the following additional term in the action

$$
S_{\mathrm{GF}}=\int d^{4} x\left(\partial^{\rho} h_{\rho \alpha}-\frac{1}{2} \partial_{\alpha} h\right)^{2},
$$

the Feynman rules of this theory can be unambiguously derived (they can be found for example in ref. [20]), thus allowing for the diagrammatic computation of the scattering amplitudes.

The relevant process for the derivation of the potential is a scattering process involving only matter fields, i.e. of the type

$$
\phi_{1} \phi_{2} \rightarrow \phi_{1} \phi_{2}
$$

with one or more gravitons in the internal propagators. Not all the terms in the amplitudes relative to this process are needed, but only those that encode a long-range and classical

\footnotetext{
${ }^{4}$ Even for relatively compact objects such as neutron stars, finite-size effects $[15,16]$ start to be important [19].

${ }^{5}$ The extension of this approach to Kerr black holes requires the inclusion of spin effects [17, 18].
} 
interaction. More precisely, the long-range condition is satisfied only when a pole in $q^{2}$ is present, where $q$ is the momentum transfer. Analytical terms in $\mathbf{q}$ would provide local or ultra-local contributions to the potential, namely proportional to a Dirac delta or its derivatives. The classical requirement is somewhat more subtle and entails selecting those components that depend on the dimensionless ratios $m_{1,2} / \sqrt{q}$. This is due to a compelling cancellation of $\hbar$ that arises in the loop expansion of processes involving an interplay between massive and massless particles, whenever the aforementioned ratio is present. For more details on this $\hbar$ cancellation we refer to the thorough analysis of ref. [21].

These conditions greatly simplify the task of computing the required classical contributions of the quantum amplitude. Moreover, they enable the use of modern techniques for the evaluation of amplitudes in the spinor-helicity formalism, whose foundations are generalized unitarity [22] and double-copy relations [23]. In this scheme, gravity tree amplitudes are obtained from the simpler gauge-theory ones and then they are employed to evaluate the singular part of the corresponding loop amplitudes. In ref. [9] such methods have been used to compute gravity amplitudes up to two loops.

The specific link between those amplitudes and the potential can be established through the following equivalent procedures:

- Matching order by order the on-shell amplitudes of the full theory discussed above with those that come from an effective field theory of two NR scalar fields $A$ and $B$ described by the Lagrangian

$$
\begin{aligned}
\mathcal{L}=\int \frac{d^{3} \boldsymbol{k}}{(2 \pi)^{3}}\left[A ^ { \dagger } ( - \boldsymbol { k } ) \left(i \partial_{t}-\right.\right. & \left.\sqrt{\boldsymbol{k}^{2}+m_{A}^{2}}\right) A(\boldsymbol{k}) \\
& \left.+B^{\dagger}(-\boldsymbol{k})\left(i \partial_{t}-\sqrt{\boldsymbol{k}^{2}+m_{B}^{2}}\right) B(\boldsymbol{k})\right] \\
& +\int \frac{d^{3} \boldsymbol{k}}{(2 \pi)^{3}} \frac{d^{3} \boldsymbol{k}^{\prime}}{(2 \pi)^{3}} V\left(\boldsymbol{k}, \boldsymbol{k}^{\prime}\right) A^{\dagger}(\boldsymbol{k}) A\left(\boldsymbol{k}^{\prime}\right) B^{\dagger}\left(-\boldsymbol{k}^{\prime}\right) B(-\boldsymbol{k})
\end{aligned}
$$

where $V\left(\boldsymbol{k}, \boldsymbol{k}^{\prime}\right)$ is the sought potential. This was originally proposed in ref. [4], in the first PM potential derivation from scattering amplitudes, and then in ref. [9] it has been employed to compute up to the $3 \mathrm{PM}$ potential.

- Adapting the Born series formalism, that is commonly used in NR quantum mechanics, to the relativistic case. Under the conditions at hand for the considered scattering process, this generalization can be easily accomplished. If we label the initial and final scattering states as $\left|p_{1}, p_{3}\right\rangle$ and $\left|p_{2}, p_{4}\right\rangle$ respectively, the Born series that gives the momentum space potential reads

$$
\begin{aligned}
\left\langle p_{2}, p_{4}|V| p_{1}, p_{3}\right\rangle= & M\left(p_{1}, p_{2}, p_{3}, p_{4}\right) \\
& -\lim _{\varepsilon \rightarrow 0^{+}} \int \frac{d^{3} \boldsymbol{k}_{1}}{(2 \pi)^{3}} \frac{d^{3} \boldsymbol{k}_{2}}{(2 \pi)^{3}} \frac{M\left(p_{1}, p_{3}, k_{1}, k_{2}\right) M\left(k_{1}, k_{2}, p_{2}, p_{4}\right)}{E_{\boldsymbol{p}_{1}}+E_{\boldsymbol{p}_{3}}-E_{\boldsymbol{k}_{1}}-E_{\boldsymbol{k}_{2}}+i \varepsilon}+\ldots,
\end{aligned}
$$

where $M$ is the full on-shell amplitude of the process (2.6) and $k_{i}=\left(E_{\boldsymbol{k}_{i}} / c, \boldsymbol{k}_{i}\right)$. Then the potential at any order $N$ in the PM expansion can be determined by extracting 
the $O\left(G^{N}\right)$ contributions in the right hand side of eq. (2.8), according to the simple correspondence

$$
N \text {-loop amplitude } \Longleftrightarrow O\left(G^{N+1}\right) \text { contributions. }
$$

This method was first introduced in ref. [5] and used to compute the potential up to the $2 \mathrm{PM}$ order.

Indeed in both these schemes the amplitudes are needed only in their long-range and classical parts. Finally, the resulting potential in momentum space can be recasted in its usual position space expression with a Fourier integration on $\boldsymbol{q}$, the spatial component of the momentum transfer.

Indeed one remarkable degree of freedom in this whole computation is represented by the invariance of the on-shell amplitudes we deal with under linearized diffeomorphisms, and mainly manifests itself as freedom in the gauge fixing choice, in principle not limited to the usual De Donder one (2.5) we also adopted. Nevertheless, even after the gauge fixing, the potential turns out to be not uniquely determined: as we will elaborate on in the following sections, the precise way in which one performs the aforementioned Fourier transform can still result in different expressions for the potential. This residual arbitrariness is specifically the one upon which our analysis is based. Finally we wish to remark that, since the potential belongs to the effective Hamiltonian, all the forms it might assume as a result of all these discretionary choices should always be connected by canonical transformations.

\subsection{The 1PM potential}

Let us now focus on the 1PM potential. In this approximation the link amplitudes-potential is quite simple: the long-range, classical part of the tree-level amplitude corresponds directly to the momentum space expression of the potential. From a diagrammatic point of view, since there are no annihilation channels to deal with, this tree amplitude consists of a single Feynman diagram that one can readily evaluate:

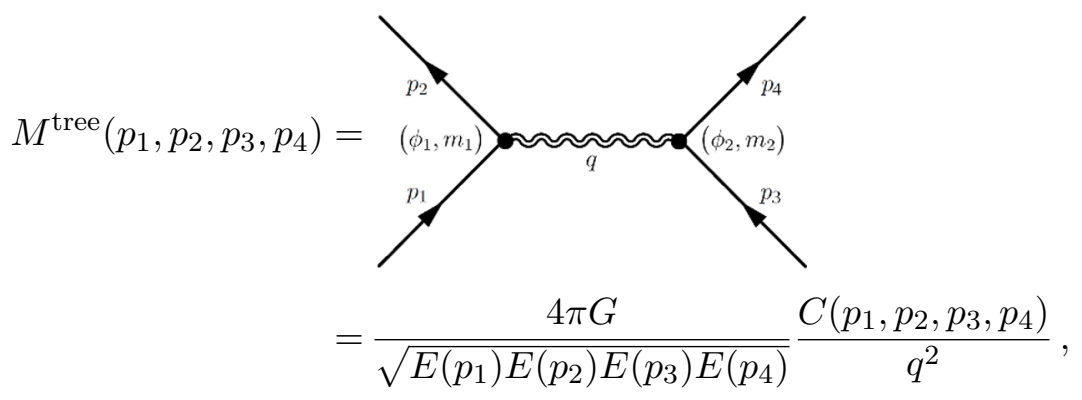

where the contraction $C$ between the pair of scalar-graviton vertices and the numerator of the graviton propagator turns out to be

$$
\begin{aligned}
C\left(p_{1}, p_{2}, p_{3}, p_{4}\right)= & \left(p_{1} \cdot p_{3}\right)\left(p_{2} \cdot p_{4}\right)+\left(p_{1} \cdot p_{4}\right)\left(p_{2} \cdot p_{3}\right)-\left(p_{1} \cdot p_{2}\right)\left(p_{3} \cdot p_{4}\right) \\
& +\left(p_{3} \cdot p_{4}\right) m_{1}^{2} c^{2}+\left(p_{1} \cdot p_{2}\right) m_{2}^{2} c^{2}-2 m_{1}^{2} m_{2}^{2} c^{4} .
\end{aligned}
$$

The next step is the Fourier transform that leads to the position space potential. Such Fourier transform is not only sensitive to what reference frame is chosen but also, and 
perhaps most importantly, on how the $\boldsymbol{q}$-dependence is distributed in the 4-momenta and energies of the particles involved in the scattering. Given its already mentioned importance for our purposes, we will proceed by carefully working out these details.

In the standard computation the Fourier transform is carried out in the center of mass reference frame with the momentum transfer defined through:

$$
q^{\mu} \equiv\left(p_{1}\right)^{\mu}-\left(p_{2}\right)^{\mu}=\left(p_{4}\right)^{\mu}-\left(p_{3}\right)^{\mu}=(0, \boldsymbol{q}),
$$

with a zero time component compatible with energy conservation. With this choice the denominator of the graviton propagator becomes simply

$$
\frac{1}{q^{2}}=-\frac{1}{|\boldsymbol{q}|^{2}} .
$$

At the same time, external 4-momenta are written as

$$
\begin{array}{ll}
\left(p_{1,3}\right)^{\mu}=\left(E_{1,2}, \pm \boldsymbol{p}\right), & \text { [incoming] } \\
\left(p_{2,4}\right)^{\mu}=\left(E_{1,2}, \pm \boldsymbol{p}^{\prime}\right), & \text { [outgoing] }
\end{array}
$$

where

$$
E_{1,2}=c \sqrt{\boldsymbol{p}^{2}+m_{1,2}^{2} c^{2}}, \quad \boldsymbol{p}^{\prime}=\boldsymbol{p}-\boldsymbol{q} .
$$

In other words the dependence of the external 3-momenta on $\boldsymbol{q}$, which then reflects itself on 4-momenta and energies, is limited to the final states:

$$
\left\{\begin{array}{l}
\boldsymbol{p}_{1}=-\boldsymbol{p}_{3}=\boldsymbol{p} \\
\boldsymbol{p}_{2}=-\boldsymbol{p}_{4}=\boldsymbol{p}-\boldsymbol{q}
\end{array} .\right.
$$

As we will see, even remaining in the center of mass reference frame this is not the only possible choice of momenta. Under these conditions one has

$$
\left[M^{\text {tree }}(\boldsymbol{q})\right]_{\substack{\text { long-range } \\ \text { classical }}}=\frac{4 \pi G}{E_{1} E_{3}} \frac{m_{1}^{2} m_{2}^{2} c^{4}-2\left(p_{1} \cdot p_{3}\right)^{2}}{|\boldsymbol{q}|^{2}} .
$$

The only $\boldsymbol{q}$-dependent quantity left is the absolute square in the denominator, so that the simple Fourier transform

$$
\int \frac{d^{3} \boldsymbol{q}}{(2 \pi)^{3}} \frac{e^{-i \boldsymbol{q} \cdot \boldsymbol{r}}}{|\boldsymbol{q}|^{2}}=\frac{1}{4 \pi r}
$$

gives directly the position space $1 \mathrm{PM}$ potential

$$
V_{1 \mathrm{PM}}^{[\text {lit.] }}(\boldsymbol{p}, \boldsymbol{r})=-\frac{G}{E_{1} E_{2}} \frac{2\left(p_{1} \cdot p_{3}\right)^{2}-m_{1}^{2} m_{2}^{2} c^{4}}{r},
$$

where, with respect to the binary system of astrophysical bodies, we can regard $\boldsymbol{p}$ as their momentum in the center of mass frame and $r$ as their relative separation. This is the 1PM result for the potential on which refs. [4, 5, 9] agree upon. 


\subsection{Issues with the NR limit of the PM potential}

The 1PM potential (2.18) contains all orders of $1 / c^{2}$ in the $c \rightarrow \infty$ expansion while being at the first order in the Newton's constant $G$. Therefore, one would expect its NR expansion should reproduce all the $O(G)$ terms of the potential encompassed by the PN Hamiltonian currently used in gravitational wave astronomy [24, 25]. At present the PN Hamiltonian has been computed up to 5.5PN order [26].

However, if we take the result (2.18) and evaluate its NR expansion up to order $O\left(1 / c^{2}\right)$ we find

$$
V_{1 \mathrm{PM}}^{[\mathrm{lit} .]}(\boldsymbol{p}, \boldsymbol{r})=-\frac{G m_{1} m_{2}}{r}-\frac{G m_{1} m_{2}}{2 r c^{2}}\left(3 \frac{\boldsymbol{p}^{2}}{m_{1}^{2}}+3 \frac{\boldsymbol{p}^{2}}{m_{2}^{2}}+8 \frac{\boldsymbol{p}^{2}}{m_{1} m_{2}}\right)+O\left(1 / c^{4}\right) .
$$

We can then compare this result to the well-know 1PN potential, first found by EinsteinInfeld-Hoffmann in ref. [8], namely

$$
V_{1 \mathrm{PN}}=-\frac{G m_{1} m_{2}}{r}-\frac{G m_{1} m_{2}}{2 r c^{2}}\left[\frac{3 \boldsymbol{p}^{2}}{m_{1}^{2}}+\frac{3 \boldsymbol{p}^{2}}{m_{2}^{2}}+\frac{7 \boldsymbol{p}^{2}}{m_{1} m_{2}}+\frac{(\boldsymbol{p} \cdot \hat{\boldsymbol{r}})^{2}}{m_{1} m_{2}}\right]+O\left(G^{2}\right) .
$$

As it clearly appears, these two results are generally different, unless one assumes $\boldsymbol{p}$ to be directed along $\hat{\boldsymbol{r}}=\frac{r}{r}$, the radial separation direction. This apparent mismatch does not imply that the potential (2.19) is incorrect. Indeed, it is well-known that the two potentials (2.19) and (2.20) are related by a canonical coordinate remapping. More generally, it is discussed in ref. [9] how a suitable canonical transformation can establish the consistency between their 3PM potential and the 4PN one.

Nevertheless, in virtue of the theoretical possibility of finding unprecedented PN results from the NR expansion of the PM ones, it would be preferable to have PM potentials which present a priori a NR behaviour consistent with what has been already computed in the PN framework. In the following section we will show how the 1PM potential can be modified in this sense, while leaving untouched the De Donder gauge fixing.

\section{A new expression for the 1PM potential}

While sticking to the center of mass frame, we employ a modified expression of the four momentum transfer, still compatible with the conservation laws. Instead of $(2.11)$ we use ${ }^{6}$

$$
q=\left(\frac{\sqrt{\left(E_{1}-E_{2}\right)\left(E_{4}-E_{3}\right)}}{c}, \boldsymbol{q}\right) .
$$

Here the time component is written in a form that satisfies in general energy conservation and that is symmetric with respect to the pair of particles involved in the scattering process. The denominator of the graviton propagator (2.12) becomes

$$
\frac{1}{q^{2}}=\frac{1}{q_{0}^{2}-|\boldsymbol{q}|^{2}}=\frac{c^{2}}{\left[E\left(p_{1}\right)-E\left(p_{2}\right)\right]\left[E\left(p_{4}\right)-E\left(p_{3}\right)\right]-|\boldsymbol{q}|^{2} c^{2}} .
$$

\footnotetext{
${ }^{6}$ This is a particular case of the generalized propagator introduced by Hiida and Okamura in ref. [27], and later taken into account also in ref. [28]. According to their notation, we are considering the propagator which follows after having fixed the gauge parameter $\mathrm{x}$ to 1 .
} 
Surprisingly, this is enough to significantly change the expression of the long-range classical part of the tree level amplitude. In fact, if we specify the 3-momenta as in (2.15), we end up with

$$
\left[M^{\text {tree }}(\boldsymbol{q})\right]_{\substack{\text { long-range } \\ \text { classical }}}=\frac{4 \pi G}{|\boldsymbol{q}|^{2}} \frac{m_{1}^{2} m_{2}^{2} c^{4}-2\left(p_{1} \cdot p_{3}\right)^{2}}{E_{1} E_{3}} \frac{1}{\frac{(\boldsymbol{p} \cdot \hat{\boldsymbol{q}})^{2}}{E_{1} E_{3}}+1}+\frac{A\left(\boldsymbol{p}^{2},(\boldsymbol{p} \cdot \boldsymbol{q})\right)}{|\boldsymbol{q}|},
$$

where $\hat{\boldsymbol{q}}=\frac{\boldsymbol{q}}{q}$. In the second term on the right hand side of (3.3) $A$ is a function of $\boldsymbol{p}^{2}$ and $(\boldsymbol{p} \cdot \boldsymbol{q})$ is explicitly given in appendix A. By checking its expression in the NR limit one discovers a series of terms proportional to odd powers of $(\boldsymbol{p} \cdot \boldsymbol{q})$. These, together with the $|\boldsymbol{q}|$ in the denominator, lead to ratios that once Fourier transformed result in imaginary terms. For example at order $O\left(1 / c^{2}\right)$, a contribution of the following type occurs

$$
\int \frac{d^{3} \boldsymbol{q}}{(2 \pi)^{3}} \frac{\boldsymbol{p} \cdot \boldsymbol{q}}{\boldsymbol{q}^{2}} e^{-i \boldsymbol{q} \cdot \boldsymbol{r}}=i \frac{\boldsymbol{p} \cdot \boldsymbol{r}}{4 \pi r^{3}}
$$

The resulting imaginary terms are not surprising in a scattering amplitude, but they cannot be accepted as parts of a classical potential. We then have to look for a choice of the momenta alternative to (2.15) that could solve this issue. To this end we repeat the computation above in a generically parameterised center of mass reference frame:

$$
\left\{\begin{array}{l}
\boldsymbol{p}_{1}=-\boldsymbol{p}_{3}=\boldsymbol{p}+a \boldsymbol{q} \\
\boldsymbol{p}_{2}=-\boldsymbol{p}_{4}=\boldsymbol{p}+(a-1) \boldsymbol{q}
\end{array}\right.
$$

where $a$ is a free real parameter. In doing so, we retrieve the amplitude (3.3) but with a difference: an overall factor $(2 a-1)$ in front of the term proportional to $A$. Clearly this means that we can get rid of all the imaginary terms mentioned above by simply setting $a=1 / 2$. Therefore the final expression for the 3 -momenta is

$$
\left\{\begin{array}{l}
\boldsymbol{p}_{1}=-\boldsymbol{p}_{3}=\boldsymbol{p}+\boldsymbol{q} / 2 \\
\boldsymbol{p}_{2}=-\boldsymbol{p}_{4}=\boldsymbol{p}-\boldsymbol{q} / 2
\end{array}\right.
$$

with the $q$-dependence now equally distributed to initial and final momenta. Accordingly, the new amplitude reads

$$
\left[M^{\text {tree }}(\boldsymbol{q})\right]_{\substack{\text { long-range } \\ \text { classical }}}=\frac{4 \pi G}{|\boldsymbol{q}|^{2}}\left[\frac{m_{1}^{2} m_{2}^{2} c^{4}-2\left(p_{1} \cdot p_{3}\right)^{2}}{E_{1} E_{3}} \frac{1}{\frac{(p \cdot \hat{q})^{2}}{E_{1} E_{3}}+1}\right]_{|\boldsymbol{q}|=0} .
$$

This is precisely the first term of (3.3), where now the quantities inside the square brackets are evaluated at $|\boldsymbol{q}|=0$ since in the new reference frame, unlike in the previous one, 4-momenta and energies actually depend on $\boldsymbol{q}$.

As usual the next step is to Fourier transform. Aside from all the $\boldsymbol{q}$-independent quantities that can be moved outside the integral, this means to evaluate

$$
\int \frac{d^{3} \boldsymbol{q}}{(2 \pi)^{3}} \frac{1}{|\boldsymbol{q}|^{2}} \frac{e^{-i \boldsymbol{q} \cdot \boldsymbol{r}}}{1+\alpha(\boldsymbol{p} \cdot \hat{\boldsymbol{q}})^{2}}
$$


where for the sake of compactness we have defined:

$$
\alpha \equiv \frac{c^{2}}{\left[E\left(p_{1}\right) E\left(p_{3}\right)\right]_{|\boldsymbol{q}|=0}}=\frac{1}{\sqrt{\boldsymbol{p}^{2}+m_{1}^{2} c^{2}} \sqrt{\boldsymbol{p}^{2}+m_{2}^{2} c^{2}}} .
$$

Then, by noting that

$$
\alpha(\boldsymbol{p} \cdot \hat{\boldsymbol{q}})^{2}=\frac{(\boldsymbol{p} \cdot \hat{\boldsymbol{q}})^{2}}{\sqrt{\boldsymbol{p}^{2}+m_{1}^{2} c^{2}} \sqrt{\boldsymbol{p}^{2}+m_{2}^{2} c^{2}}} \leq \frac{|\boldsymbol{p}|^{2}}{\sqrt{\boldsymbol{p}^{2}+m_{1}^{2} c^{2}} \sqrt{\boldsymbol{p}^{2}+m_{2}^{2} c^{2}}}<1
$$

we can rewrite the integrand in (3.8) using

$$
\frac{1}{1+\alpha(\boldsymbol{p} \cdot \hat{\boldsymbol{q}})^{2}}=\sum_{n=0}^{+\infty}(-1)^{n} \alpha^{n}(\boldsymbol{p} \cdot \hat{\boldsymbol{q}})^{2 n}
$$

and evaluate the Fourier transform term by term:

$$
\left[\frac{(\boldsymbol{p} \cdot \hat{\boldsymbol{q}})^{2 n}}{|\boldsymbol{q}|^{2}}\right]_{\mathrm{FT}} \equiv \int \frac{d^{3} \boldsymbol{q}}{(2 \pi)^{3}} \frac{(\boldsymbol{p} \cdot \hat{\boldsymbol{q}})^{2 n}}{|\boldsymbol{q}|^{2}} e^{-i \boldsymbol{q} \cdot \boldsymbol{r}}=\frac{1}{4 \pi r} \frac{(2 n) !}{2^{2 n}(n !)^{2}}\left[\boldsymbol{p}^{2}-(\boldsymbol{p} \cdot \hat{\boldsymbol{r}})^{2}\right]^{n},
$$

for a non-negative integer $n$. In this way we find

$$
\begin{aligned}
\int \frac{d^{3} \boldsymbol{q}}{(2 \pi)^{3}} \frac{1}{|\boldsymbol{q}|^{2}} \frac{e^{-i \boldsymbol{q} \cdot \boldsymbol{r}}}{1+\alpha(\boldsymbol{p} \cdot \hat{\boldsymbol{q}})^{2}} & =\frac{1}{4 \pi r} \sum_{n=0}^{+\infty}(-1)^{n} \frac{(2 n) !}{(n !)^{2}}\left[\alpha \frac{\boldsymbol{p}^{2}-(\boldsymbol{p} \cdot \hat{\boldsymbol{r}})^{2}}{4}\right]^{n} \\
& =\frac{1}{4 \pi r} \frac{1}{\sqrt{1+\alpha\left[\boldsymbol{p}^{2}-(\boldsymbol{p} \cdot \hat{\boldsymbol{r}})^{2}\right]}}
\end{aligned}
$$

The last equality holds since

$$
\sum_{n=0}^{+\infty}(-1)^{n} \frac{(2 n) !}{(n !)^{2}} z^{n}=\frac{1}{\sqrt{1+4 z}} \text { for }|z|<\frac{1}{4}
$$

and, just like it was shown in (3.10), we have

$$
\alpha\left[\boldsymbol{p}^{2}-(\boldsymbol{p} \cdot \hat{\boldsymbol{r}})\right]<1 .
$$

In conclusion our final result for the position space 1PM potential in the center of mass frame is

$$
V_{1 \mathrm{PM}}(\boldsymbol{p}, \boldsymbol{r})=-\frac{G}{r}\left[\frac{2\left(p_{1} \cdot p_{3}\right)^{2}-m_{1}^{2} m_{2}^{2} c^{4}}{E\left(p_{1}\right) E\left(p_{3}\right)} \frac{1}{\sqrt{1+\frac{c^{2}}{E\left(p_{1}\right) E\left(p_{3}\right)}\left[\boldsymbol{p}^{2}-(\boldsymbol{p} \cdot \hat{\boldsymbol{r}})^{2}\right]}}\right]_{|\boldsymbol{q}|=0} .
$$

Interestingly, we can rewrite (3.16) as

$$
V_{1 \mathrm{PM}}^{\text {new }}=V_{1 \mathrm{PM}}^{\text {lit. }} \times\left(1+\frac{\boldsymbol{p}^{2}-(\boldsymbol{p} \cdot \hat{\boldsymbol{r}})^{2}}{\sqrt{\boldsymbol{p}^{2}+m_{1}^{2} c^{2}} \sqrt{\boldsymbol{p}^{2}+m_{2}^{2} c^{2}}}\right)^{-\frac{1}{2}}
$$


where $V_{1 \mathrm{PM}}^{\text {lit. }}$ is the known $1 \mathrm{PM}$ potential (2.18). Moreover, thanks to the dimensionless additional factor multiplying $V_{1 \mathrm{PM}}^{\text {lit. }}$, the NR expansion now gives

$$
V_{1 \mathrm{PM}}(\boldsymbol{p}, \boldsymbol{r})=-\frac{G m_{1} m_{2}}{r}-\frac{G m_{1} m_{2}}{2 r c^{2}}\left[\frac{3 \boldsymbol{p}^{2}}{m_{1}^{2}}+\frac{3 \boldsymbol{p}^{2}}{m_{2}^{2}}+\frac{7 \boldsymbol{p}^{2}}{m_{1} m_{2}}+\frac{(\boldsymbol{p} \cdot \hat{\boldsymbol{r}})^{2}}{m_{1} m_{2}}\right]+O\left(1 / c^{4}\right)
$$

which exactly reproduces the $O(G)$ terms of the 1PN Einstein-Infeld-Hoffmann potential (2.20). This is enough to reestablish the matching with the 1PN potential as a whole, since the static term

$$
\frac{G^{2} m_{1} m_{2}\left(m_{1}+m_{2}\right)}{2 r^{2} c^{2}}
$$

is already correctly reproduced by every $2 \mathrm{PM}$ potential derived with the scattering amplitude method in the literature $[4,5,9]$. The new 1PM potential (3.16) represents the main result of this Letter.

\section{NR limit beyond the 1PN order}

The extension of this convenient direct match between the NR limit of the 1PM potential (3.16) and the PN results available in the literature to include higher order in the PN expansion is not however immediately obvious and requires further investigation.

From ref. [29], the paper which first presented a 2PN two-body Hamiltonian, we can extract the $O\left(G / c^{4}\right)$ component of the $2 \mathrm{PN}$ potential in the center of mass frame, which is given by

$$
V_{2 \mathrm{PN}}^{\left[O\left(G / c^{4}\right)\right]}=\frac{G m_{1} m_{2}}{8 r c^{4}}\left(\frac{5 \boldsymbol{p}^{4}}{m_{1}^{4}}-\frac{13 \boldsymbol{p}^{4}}{m_{1}^{2} m_{2}^{2}}-\frac{3(\boldsymbol{p} \cdot \hat{\boldsymbol{r}})^{4}}{m_{1}^{2} m_{2}^{2}}-\frac{2 \boldsymbol{p}^{2}(\boldsymbol{p} \cdot \hat{\boldsymbol{r}})^{2}}{m_{1}^{2} m_{2}^{2}}+\frac{5 \boldsymbol{p}^{4}}{m_{2}^{4}}\right) .
$$

By comparing the non-relativistic expansion of our 1PM potential with (4.1), the following discrepancy arises:

$$
\begin{aligned}
D_{4} \equiv V_{1 \mathrm{PM}}^{\left[O\left(G / c^{4}\right)\right]}-V_{2 \mathrm{PN}}^{\left[O\left(G / c^{4}\right)\right]} & =\frac{G}{2 c^{4} r}\left(\frac{1}{m_{1}}+\frac{1}{m_{2}}\right)^{2}\left[\boldsymbol{p}^{4}-\boldsymbol{p}^{2}(\boldsymbol{p} \cdot \hat{\boldsymbol{r}})^{2}\right] \\
& =\frac{4 \pi G}{c^{4}}\left(\frac{1}{m_{1}}+\frac{1}{m_{2}}\right)^{2}\left[\frac{(\boldsymbol{p} \cdot \hat{\boldsymbol{q}})^{2}}{|\boldsymbol{q}|^{2}}\right]_{\mathrm{FT}} \boldsymbol{p}^{2} .
\end{aligned}
$$

We haven't yet been able to find any obvious adjustment of our 1PM potential such that the discrepancy (4.2) end up being resolved. Very likely, since PN results are not found in the harmonic gauge, here the problem lies in the gauge fixing choice. We discuss this issue further in section 6 .

What we could do for now is to check whether and how the correct NR limit of our 1PM potential could be adjusted by performing some a posteriori canonical transformation. We have done this explicitly for the $2 \mathrm{PN}$ order only, but the same procedure could be likely extended to also ensure the matching at higher PN orders. Generally speaking, such a transformation should not be restricted to the potential only, but extended also to the other component of our effective Hamiltonian, the free-particle one. The latter is precisely the part of the Hamiltonian which could in principle provide the desired correction. Indeed, since our Hamiltonian reproduces the expected NR limit up to the 1PN order, the transformation we look for has to be proportional, at least, to $G / c^{4}$ : 
- if a factor $G$ is not included, the NR limit of the free-particle energy results is unavoidably spoiled.

- if at least a factor $1 / c^{4}$ is not included, the Newtonian and the $1 \mathrm{PN}$ parts of the potential are spoiled.

Therefore the additional $O(G)$ terms that we need in order to fix the NR behaviour of our potential could only come from the canonically transformed $O\left(G^{0}\right)$ part of our Hamiltonian. Bearing this in mind, we start with a parameterized coordinate transformation defined as in section 11 of ref. [9], where the new coordinates are expressed in terms of the old ones through powers of $\boldsymbol{p}^{2}$ and $(\boldsymbol{p} \cdot \hat{\boldsymbol{r}})$ times free functions of the masses. Actually, to fix the matching at the level of the $2 \mathrm{PN}$ we can limit ourselves to the contributions proportional to $G$ and with powers of the momentum compatible with the $2 \mathrm{PN}$ order. Then we find the constraints on the functions of the masses that enstablish the validity of the fundamental poisson bracket relations in the new coordinates, so as to ensure canonicity for our transformation. At last, we fix the remaining free functions so that the resulting transformation removes the discrepancy (4.2). The resulting canonical transform is

$$
\left\{\begin{array}{l}
\boldsymbol{p} \rightarrow\left[1-\frac{G}{2 r c^{4}}\left(\frac{1}{m_{1}}+\frac{1}{m_{2}}\right) \boldsymbol{p}^{2}\right] \boldsymbol{p}+\left[\frac{G}{2 r c^{4}}\left(\frac{1}{m_{1}}+\frac{1}{m_{2}}\right)(\boldsymbol{p} \cdot \hat{\boldsymbol{r}}) \boldsymbol{p}^{2}\right] \boldsymbol{r} \\
\boldsymbol{r} \rightarrow\left[1-\frac{G}{2 r c^{4}}\left(\frac{1}{m_{1}}+\frac{1}{m_{2}}\right) \boldsymbol{p}^{2}\right] \boldsymbol{r}+\left[\frac{G}{c^{4}}\left(\frac{1}{m_{1}}+\frac{1}{m_{2}}\right)(\boldsymbol{p} \cdot \hat{\boldsymbol{r}})\right] \boldsymbol{p}
\end{array}\right.
$$

The existence of such canonical transformation provides a first non-trivial check for our new $1 \mathrm{PM}$ potential. The procedure to fix the matching with the $3 \mathrm{PN}$ and $4 \mathrm{PN}$ potentials is essentially the same, as long as the starting transformation is also equipped with those contributions that are compatible with the $3 \mathrm{PN}$ and $4 \mathrm{PN}$ orders.

\section{$5 \quad 1 P M$ scattering angle with the new potential}

Starting from an effective Hamiltonian one can always obtain physical observables. Indeed they must remain unaffected under any non-physical modification, thus providing the perfect context to test the physical consistency of our newly found expression for the $1 \mathrm{PM}$ potential given in eq. (3.16), compared to the one normally used in the literature. Along these lines, in this section we will compute the 1PM scattering angle from our potential (3.16) and show that the resulting expression is consistent with the known scattering angle that can be found in the literature $[10-14,30]$.

\subsection{The scattering angle}

Since in our setup the spin is not involved, we can restrict ourselves to the case where the motion is lying on a plane. Accordingly, the momentum can be decomposed as

$$
\boldsymbol{p}^{2}=\boldsymbol{p}_{r}^{2}+\frac{\boldsymbol{L}^{2}}{r^{2}}
$$


in terms of the radial momentum $\boldsymbol{p}_{r}$ and the conserved angular momentum $\boldsymbol{L}$. Once $\boldsymbol{p}_{r}$ is known, the scattering angle follows from the formula $[5,31]$ :

$$
\chi=-\pi-2 \int_{r_{\min }}^{+\infty} d r \frac{\partial p_{r}}{\partial L}
$$

where $r_{\min }$ corresponds to the minimum distance between the two scattering bodies. Denoting with $E$ the total conserved energy, we can extract the expression for the radial momentum at order $O(G)$ by solving perturbatively the equation

$$
H(\boldsymbol{r}, \boldsymbol{p})=c \sqrt{\boldsymbol{p}^{2}+m_{1}^{2}}+c \sqrt{\boldsymbol{p}^{2}+m_{2}^{2}}+V_{1 \mathrm{PM}}\left(\boldsymbol{r}, \boldsymbol{p}^{2}\right)=E \quad \text { (Energy Conservation) }
$$

together with the momentum decomposition (5.1). In this way we find

$$
p_{r}^{2}(r, L)=p_{\infty}^{2}-\frac{L^{2}}{r^{2}}+P^{(1)} \frac{G}{r}+O\left(G^{2}\right)
$$

where we defined the momentum at infinity, $p_{\infty}$, such that

$$
E=c \sqrt{p_{\infty}^{2}+m_{1}^{2}}+c \sqrt{p_{\infty}^{2}+m_{2}^{2}}
$$

and the 1PM coefficient can be derived from our expression for the 1PM potential (3.16) and is given by

$$
\begin{aligned}
P^{(1)} \equiv & \left.\frac{4\left(p_{\infty}^{2}+\sqrt{p_{\infty}^{2}+m_{1}^{2} c^{2}} \sqrt{p_{\infty}^{2}+m_{2}^{2} c^{2}}\right)^{2}-2 m_{2}^{2} m_{2}^{2} c^{4}}{E^{2}}\right)^{-\frac{1}{2}} \\
& \times\left(1+\frac{L^{2}}{\sqrt{p_{\infty}^{2}+m_{1}^{2} c^{2}} \sqrt{p_{\infty}^{2}+m_{2}^{2} c^{2}} r^{2}}\right.
\end{aligned}
$$

We note that at this stage the new potential (3.16) modifies only the expression of $P^{(1)}$ which differs from the one used in the literature just for the multiplicative adimensional factor we isolated in the second line of (5.6). Nevertheless, this difference has a remarkable impact in the computation of the scattering angle (5.2) because the new factor features both an $L$ and an $r$ dependence. The first one is relevant for the integrand in (5.2),

$$
\frac{\partial p_{r}}{\partial L}=-\frac{L^{2}}{r^{2} p_{r}(r)}+\frac{G}{2 r p_{r}(r)} \frac{\partial P^{(1)}}{\partial L}
$$

while the $r$ dependence affects the position of the inversion point $r_{\min }$. In fact the condition

$$
p_{r}\left(r_{\min }\right)=0,
$$

from which $r_{\min }$ was usually derived as an exact solution, can now be properly solved only through a perturbative treatment similar to the one adopted for eq. (5.3). At order $G$ this procedure allows to find

$$
r_{\min }=r_{0}-G \frac{r_{0} P_{\infty}^{(1)}}{2 p_{\infty}^{2} \sqrt{r_{0}^{2}+\left(\alpha_{\infty} L\right)^{2}}}
$$


compactly written in terms of

$$
\begin{aligned}
r_{0} & \equiv \frac{L}{p_{\infty}}, \quad P_{\infty}^{(1)} \equiv \frac{4\left(p_{\infty}^{2}+\sqrt{p_{\infty}^{2}+m_{1}^{2} c^{2}} \sqrt{p_{\infty}^{2}+m_{2}^{2} c^{2}}\right)^{2}-2 m_{2}^{2} m_{2}^{2} c^{4}}{E^{2}} \\
\alpha_{\infty} & \equiv \frac{1}{\sqrt{p_{\infty}^{2}+m_{1}^{2} c^{2}} \sqrt{p_{\infty}^{2}+m_{2}^{2} c^{2}}}
\end{aligned}
$$

Using this notation and the expression of $p_{r}$ given in (5.4) we get to the following result for the scattering angle

$$
\chi=-\pi+2 r_{0} I_{1}+G r_{0} P_{\infty}^{(1)} \alpha_{\infty} I_{2}
$$

where the integrals

$$
I_{1} \equiv \int_{r_{\min }}^{+\infty} d r \frac{1}{r \sqrt{r^{2}-r_{0}^{2}+G \frac{P_{\infty}^{(1)} r^{2}}{p_{\infty}^{2} \sqrt{r^{2}+\left(\alpha_{\infty} L\right)^{2}}}}}
$$

and

$$
I_{2} \equiv \int_{r_{\min }}^{+\infty} d r \frac{r}{\left(r^{2}+\left(\alpha_{\infty} L\right)^{2}\right)^{\frac{3}{2}} \sqrt{r^{2}-r_{0}^{2}+G \frac{P_{\infty}^{(1)} r^{2}}{p_{\infty}^{2} \sqrt{r^{2}+\left(\alpha_{\infty} L\right)^{2}}}}}
$$

have to be evaluated respectively at order $O(G)$ and $O\left(G^{0}\right)$.

In both these computations we used

$$
\int_{g(G)}^{+\infty} d r f(r, G)=\int_{g(0)}^{+\infty} d r f(r, 0)+\left.G\left(\frac{\partial}{\partial G} \int_{g(G)}^{+\infty} d r f(r, G)\right)\right|_{G=0}+O\left(G^{2}\right)
$$

and

$$
\frac{\partial}{\partial G} \int_{g(G)}^{+\infty} d r f(r, G)=-f(g(G), G) \frac{\partial}{\partial G} g(G)+\int_{g(G)}^{+\infty} d r \frac{\partial}{\partial G} f(r, G) .
$$

Carefully handling a couple of divergent terms for $r=r_{0}$ that arise in the evaluation of $I_{1}$ and ultimately cancel each other out, we find

$$
\begin{aligned}
& I_{1}=\frac{\pi}{2 r_{0}}+G \frac{P_{\infty}^{(1)}}{2 p_{\infty}^{2}\left(r_{0}^{2}+\left(\alpha_{\infty} L\right)^{2}\right)}+O\left(G^{2}\right) \\
& I_{2}=\frac{1}{r_{0}^{2}+\left(\alpha_{\infty} L\right)^{2}}+O(G) .
\end{aligned}
$$

Therefore our final result for the scattering angle is

$$
\chi=\frac{G P_{\infty}^{(1)} r_{0}}{p_{\infty}^{2}\left(r_{0}^{2}+\left(\alpha_{\infty} L\right)^{2}\right)}+\frac{G P_{\infty}^{(1)} r_{0} \alpha_{\infty}^{2}}{r_{0}^{2}+\left(\alpha_{\infty} L\right)^{2}}+O\left(G^{2}\right)=\frac{G P_{\infty}^{(1)}}{L p_{\infty}}+O\left(G^{2}\right)
$$

which is in perfect agreement with the one computed in refs. [10-14, 30]. 


\section{Conclusion}

In this paper we showed how the room for manoeuvre in the Fourier transform that completes the derivation of the 1PM potential from scattering amplitudes enables us to find a new expression for the 1PM potential that generalizes what was previously found in the literature. This results in an additional dimensionless factor that should be multiplied to the standard 1PM potential and that, in the non relativistic limit, directly and correctly reproduces the Einstein-Infeld-Hoffman 1PN potential.

This nice feature is not immediately enjoyed by the higher PN orders and, at this stage, we have provided the canonical transformation from which one can reproduce the $\mathrm{PN}$ potential at the $2 \mathrm{PN}$ order, starting with our new $1 \mathrm{PM}$ potential (3.16). A future goal would be to provide an expression for the PM potential which, once expanded for small velocities, gives automatically the known result in the PN expansion, without the need of performing any canonical transformation. This would indeed be an interesting task, although highly non-trivial: the PN results $[24,25,29]$ we used as a reference are all obtained in ADM coordinates. Moreover in ref. [29] it was shown that the approach that uses harmonic coordinates leads to a generalized acceleration-dependent Lagrangian, which prevents the derivation of a proper associated Hamiltonian. Therefore we believe that a possible resolution of this issue can be traced back to the gauge choice. In this respect it should be mentioned that the possibility of directly reproducing PN results beyond the first order is likely lost already when one employs the linear expanded metric (2.4). That is because such a choice limits the gauge fixing freedom from the start to just the linearized gauges, which seem unsuitable for the purpose at hand. All this considered, a resolution of this issue could require the development of an alternative quantization procedure instead of the customary one we have adopted here.

Nevertheless, by reproducing the correct 1PM scattering angle we have performed a consistency check of our extended result for the 1PM potential that shows its physical equivalence to the $1 \mathrm{PM}$ potential found previously in the literature $[5,9,10]$.

\section{Acknowledgments}

We thank S. Mezzasoma and F. Camilloni for their contribution in an early stage of this project. We thank N. E. Bjerrum-Bohr for reading the manuscript and for useful comments. T. H. acknowledges support from the Independent Research Fund Denmark grant number DFF-6108-00340 "Towards a deeper understanding of black holes with non-relativistic holography". G. G. and M. O. acknowledge support from the project "Black holes, neutron stars and gravitational waves" financed by Fondo Ricerca di Base 2018 of the University of Perugia. T. H. thanks Perugia University and G. G. and M. O. thank Niels Bohr Institute for hospitality.

\section{A The function $A$}

In this appendix we give the explicit expression for the function $A\left(\boldsymbol{p}^{2},(\boldsymbol{p} \cdot \boldsymbol{q})\right)$ that appears in eq. (3.3). We can write

$$
A\left(\boldsymbol{p}^{2},(\boldsymbol{p} \cdot \boldsymbol{q})\right)=\frac{\operatorname{Num} A}{\operatorname{Den} A}
$$


where

$$
\begin{aligned}
\operatorname{Num} A= & 2 \pi G(\boldsymbol{p} \cdot \boldsymbol{q})\left(3 m_{1}^{2} m_{2}^{2} c^{4}+2 \boldsymbol{p}^{2}\left(m_{1}^{2}+m_{2}^{2}\right) c^{2}\right) \\
& \times\left\{\left[2 m_{1}^{2} m_{2}^{2} c^{4}+\left(m_{1}^{2}+m_{2}^{2}\right) E_{1} E_{3}+2 \boldsymbol{p}^{2}\left(\frac{E_{1} E_{3}}{c^{2}}+\left(m_{1}^{2}+m_{2}^{2}\right) c^{2}\right)+2 \boldsymbol{p}^{4}\right]\right. \\
& +4(\boldsymbol{p} \cdot \boldsymbol{q})^{2}\left[\boldsymbol{p}^{4}\left(4 \frac{E_{1} E_{3}}{c^{2}}+5\left(m_{1}^{2}+m_{2}^{2}\right) c^{2}\right)+m_{1}^{2} m_{2}^{2} c^{4}\left(2 \frac{E_{1} E_{3}}{c^{2}}+\left(m_{1}^{2}+m_{2}^{2}\right) c^{2}\right)\right. \\
& \left.\left.+\boldsymbol{p}^{2}\left(\left(m_{1}^{4}+6 m_{1}^{2} m_{2}^{2}+m_{2}^{4}\right) c^{4}+3\left(m_{1}^{2}+m_{2}^{2}\right) E_{1} E_{3}\right)+4 \boldsymbol{p}^{6}\right]\right\} \\
\operatorname{Den} A= & \frac{\left(E_{1} E_{3}\right)^{2}}{c^{2}}\left(\frac{E_{1} E_{3}}{c^{2}}+(\boldsymbol{p} \cdot \boldsymbol{q})^{2}\right)^{2} .
\end{aligned}
$$

Open Access. This article is distributed under the terms of the Creative Commons Attribution License (CC-BY 4.0), which permits any use, distribution and reproduction in any medium, provided the original author(s) and source are credited.

\section{References}

[1] Y. Iwasaki, Quantum theory of gravitation vs. classical theory - Fourth-order potential, Prog. Theor. Phys. 46 (1971) 1587 [InSPIRE].

[2] L. Blanchet, Gravitational radiation from post-Newtonian sources and inspiralling compact binaries, Living Rev. Rel. 17 (2014) 2 [arXiv:1310.1528] [INSPIRE].

[3] N.E.J. Bjerrum-Bohr, P.H. Damgaard, G. Festuccia, L. Planté and P. Vanhove, General relativity from scattering amplitudes, Phys. Rev. Lett. 121 (2018) 171601 [arXiv: 1806.04920] [INSPIRE].

[4] C. Cheung, I.Z. Rothstein and M.P. Solon, From scattering amplitudes to classical potentials in the post-minkowskian expansion, Phys. Rev. Lett. 121 (2018) 251101 [arXiv:1808.02489] [INSPIRE].

[5] A. Cristofoli, N.E.J. Bjerrum-Bohr, P.H. Damgaard and P. Vanhove, Post-Minkowskian Hamiltonians in general relativity, Phys. Rev. D 100 (2019) 084040 [arXiv:1906.01579] [INSPIRE].

[6] A. Cristofoli, P.H. Damgaard, P. Di Vecchia and C. Heissenberg, Second-order post-Minkowskian scattering in arbitrary dimensions, JHEP 07 (2020) 122 [arXiv: 2003.10274] [INSPIRE].

[7] A. Antonelli, A. Buonanno, J. Steinhoff, M. van de Meent and J. Vines, Energetics of two-body Hamiltonians in post-Minkowskian gravity, Phys. Rev. D 99 (2019) 104004 [arXiv: 1901.07102] [INSPIRE].

[8] A. Einstein, L. Infeld and B. Hoffmann, The gravitational equations and the problem of motion, Annals Math. 39 (1938) 65 [INSPIRE].

[9] Z. Bern, C. Cheung, R. Roiban, C.-H. Shen, M.P. Solon and M. Zeng, Black hole binary dynamics from the double copy and effective theory, JHEP 10 (2019) 206 [arXiv: 1908.01493] [INSPIRE]. 
[10] A. Koemans Collado, P. Di Vecchia and R. Russo, Revisiting the second post-Minkowskian eikonal and the dynamics of binary black holes, Phys. Rev. D 100 (2019) 066028 [arXiv: 1904.02667] [INSPIRE].

[11] R.L. Sugar and R. Blankenbecler, Eikonal expansion, Phys. Rev. 183 (1969) 1387 [InSPIRE].

[12] A. Paliov and S. Rosendorff, Exponentially small scattering amplitude in high energy potential scattering. 2, J. Math. Phys. 15 (1974) 1045 [INSPIRE].

[13] S.J. Wallace, Eikonal expansion, Annals Phys. 78 (1973) 190 [InSPIRE].

[14] N.E.J. Bjerrum-Bohr, A. Cristofoli and P.H. Damgaard, Post-Minkowskian scattering angle in Einstein gravity, JHEP 08 (2020) 038 [arXiv: 1910.09366] [INSPIRE].

[15] W.D. Goldberger and I.Z. Rothstein, An effective field theory of gravity for extended objects, Phys. Rev. D 73 (2006) 104029 [hep-th/0409156] [INSPIRE].

[16] C. Cheung and M.P. Solon, Tidal effects in the post-Minkowskian expansion, Phys. Rev. Lett. 125 (2020) 191601 [arXiv:2006.06665] [INSPIRE].

[17] M.-Z. Chung, Y.-T. Huang, J.-W. Kim and S. Lee, The simplest massive S-matrix: from minimal coupling to Black Holes, JHEP 04 (2019) 156 [arXiv:1812.08752] [INSPIRE].

[18] A. Guevara, A. Ochirov and J. Vines, Scattering of spinning black holes from exponentiated soft factors, JHEP 09 (2019) 056 [arXiv:1812.06895] [INSPIRE].

[19] T. Hinderer, Tidal Love numbers of neutron stars, Astrophys. J. 677 (2008) 1216 [arXiv: 0711.2420] [INSPIRE].

[20] B.R. Holstein and A. Ross, Spin effects in long range gravitational scattering, arXiv:0802.0716 [INSPIRE].

[21] D.A. Kosower, B. Maybee and D. O'Connell, Amplitudes, observables, and classical scattering, JHEP 02 (2019) 137 [arXiv:1811.10950] [INSPIRE].

[22] Z. Bern, L.J. Dixon, D.C. Dunbar and D.A. Kosower, One loop $n$ point gauge theory amplitudes, unitarity and collinear limits, Nucl. Phys. B 425 (1994) 217 [hep-ph/9403226] [INSPIRE].

[23] Z. Bern, J.J.M. Carrasco and H. Johansson, Perturbative quantum gravity as a double copy of gauge theory, Phys. Rev. Lett. 105 (2010) 061602 [arXiv:1004.0476] [INSPIRE].

[24] P. Jaranowski and G. Schäfer, Derivation of local-in-time fourth post-Newtonian ADM Hamiltonian for spinless compact binaries, Phys. Rev. D 92 (2015) 124043 [arXiv: 1508.01016] [INSPIRE].

[25] T. Damour, P. Jaranowski and G. Schäfer, Nonlocal-in-time action for the fourth post-Newtonian conservative dynamics of two-body systems, Phys. Rev. D 89 (2014) 064058 [arXiv: 1401 .4548] [INSPIRE].

[26] D. Bini, T. Damour and A. Geralico, Binary dynamics at the fifth and fifth-and-a-half post-Newtonian orders, Phys. Rev. D 102 (2020) 024062 [arXiv:2003.11891] [InSPIRE].

[27] K. Hiida and H. Okamura, Gauge transformation and gravitational potentials, Prog. Theor. Phys. 47 (1972) 1743 [INSPIRE].

[28] N.E. Bjerrum-Bohr, J.F. Donoghue and B.R. Holstein, Quantum gravitational corrections to the nonrelativistic scattering potential of two masses, Phys. Rev. D 67 (2003) 084033 [Erratum ibid. 71 (2005) 069903] [hep-th/0211072] [INSPIRE]. 
[29] T. Damour and G. Schaefer, Higher order relativistic periastron advances and binary pulsars, Nuovo Cim. B 101 (1988) 127 [INSPIRE].

[30] G. Kälin and R.A. Porto, Post-Minkowskian effective field theory for conservative binary dynamics, JHEP 11 (2020) 106 [arXiv:2006.01184] [INSPIRE].

[31] T. Damour, Gravitational scattering, post-Minkowskian approximation and effective one-body theory, Phys. Rev. D 94 (2016) 104015 [arXiv:1609.00354] [InSPIRE]. 